\title{
Local integrability of strong and iterated maximal functions
}

\author{
by \\ Paul Alton Hagelstein (Princeton, NJ)
}

\begin{abstract}
Let $M_{\mathrm{S}}$ denote the strong maximal operator. Let $M_{x}$ and $M_{y}$ denote the one-dimensional Hardy-Littlewood maximal operators in the horizontal and vertical directions in $\mathbb{R}^{2}$. A function $h$ supported on the unit square $Q=[0,1] \times[0,1]$ is exhibited such that $\int_{Q} M_{y} M_{x} h<\infty$ but $\int_{Q} M_{x} M_{y} h=\infty$. It is shown that if $f$ is a function supported on $Q$ such that $\int_{Q} M_{y} M_{x} f<\infty$ but $\int_{Q} M_{x} M_{y} f=\infty$, then there exists a set $A$ of finite measure in $\mathbb{R}^{2}$ such that $\int_{A} M_{\mathrm{S}} f=\infty$.
\end{abstract}

We begin by listing some basic definitions.

Definition 1. Let $f$ be a measurable function defined on $\mathbb{R}^{n}$. Denote by $B(p, r)$ the Euclidean ball in $\mathbb{R}^{n}$ centered at $p$ of radius $r$, and by $|B(p, r)|$ the Lebesgue measure of $B(p, r)$. The Hardy-Littlewood maximal function of $f$ is defined on $\mathbb{R}^{n}$ by

$$
M_{\mathrm{HL}} f(p)=\sup _{r>0} \frac{1}{|B(p, r)|} \int_{B(p, r)}|f(z)| d z .
$$

Definition 2. Let $f$ be a measurable function defined on $\mathbb{R}^{2}$. The strong maximal function of $f$ is defined on $\mathbb{R}^{2}$ by

$$
M_{\mathrm{S}} f(x, y)=\sup _{\substack{x_{1}<x<x_{2} \\ y_{1}<y<y_{2}}} \frac{1}{\left(x_{2}-x_{1}\right)\left(y_{2}-y_{1}\right)} \int_{x_{1}}^{x_{2}} \int_{y_{1}}^{y_{2}}|f(u, v)| d v d u .
$$

Definition 3. Let $f$ be a measurable function defined on $\mathbb{R}^{2}$. The horizontal maximal function of $f$ is defined on $\mathbb{R}^{2}$ by

$$
M_{x} f(u, v)=\sup _{u_{1}<u<u_{2}} \frac{1}{u_{2}-u_{1}} \int_{u_{1}}^{u_{2}}|f(w, v)| d w .
$$

2000 Mathematics Subject Classification: Primary 42B25. 
Definition 4. Let $f$ be a measurable function defined on $\mathbb{R}^{2}$. The vertical maximal function of $f$ is defined on $\mathbb{R}^{2}$ by

$$
M_{y} f(u, v)=\sup _{v_{1}<v<v_{2}} \frac{1}{v_{2}-v_{1}} \int_{v_{1}}^{v_{2}}|f(u, w)| d w .
$$

The following Orlicz spaces will be very useful to us.

Definition 5. Let $I^{(n)}$ denote the unit n-cube $[0,1] \times \ldots \times[0,1]$ in $\mathbb{R}^{n}$. $L(\log L)^{k}\left(I^{(n)}\right)$ is the Lebesgue space of functions on $I^{(n)}$ with norm

$$
\|f\|_{L(\log L)^{k}\left(I^{(n)}\right)}=\inf \left\{c>0: \int_{I^{(n)}} \frac{|f|}{c} \log \left(3+\frac{|f|}{c}\right)^{k} \leq 1\right\}<\infty .
$$

In the following we will denote the unit square $I^{(2)}$ in $\mathbb{R}^{2}$ by $Q$. Also, the $k$-fold iteration $M_{\mathrm{HL}} \circ \ldots \circ M_{\mathrm{HL}}$ of the maximal operator $M_{\mathrm{HL}}$ will be denoted by $M_{\mathrm{HL}}^{k}$.

We now recall the following theorem of E. M. Stein:

Theorem 6 ([11]). Let $k$ be a positive integer. There exist constants $0<c=c(k, n)<C=C(k, n)<\infty$ such that if $f$ is supported on $I^{(n)}$, then

$$
c \int_{I^{(n)}} M_{\mathrm{HL}}^{k} f \leq\|f\|_{L(\log L)^{k}\left(I^{(n)}\right)} \leq C \int_{I^{(n)}} M_{\mathrm{HL}}^{k} f .
$$

Inequalities such as (6) in the case $k=1$ will often be denoted by

$$
\|f\|_{L \log L\left(I^{(n)}\right)} \sim \int_{I^{(n)}} M_{\mathrm{HL}} f
$$

for the remainder of this paper.

We now show that there exists a function $h$ supported on $Q$ such that $\int_{Q} M_{y} M_{x} h<\infty$, but $\int_{Q} M_{x} M_{y} h=\infty$.

THEOREM 7. There exists a function $h$, supported on $Q$, such that $\int_{Q} M_{x} M_{y} h=\infty$, but $\int_{Q} M_{y} M_{x} h<\infty$.

Proof. We define the functions $h_{2^{n}}$ as follows:

$$
h_{2^{n}}(x, y)=\sum_{m=0}^{2^{n}-1} 2^{2^{n}-m-1} \chi_{\left[0,2^{-2^{n}+m+1}\right]}(x) \cdot \chi_{\left[m \cdot 2^{-n},(m+1) \cdot 2^{-n}\right]}(y) .
$$

The functions $h_{2}, h_{4}$, and $h_{8}$ are depicted in Figure 1 .

Lemma 8.

$$
\int_{Q} M_{x} M_{y} h_{2^{n}} \sim n \cdot 2^{n}
$$



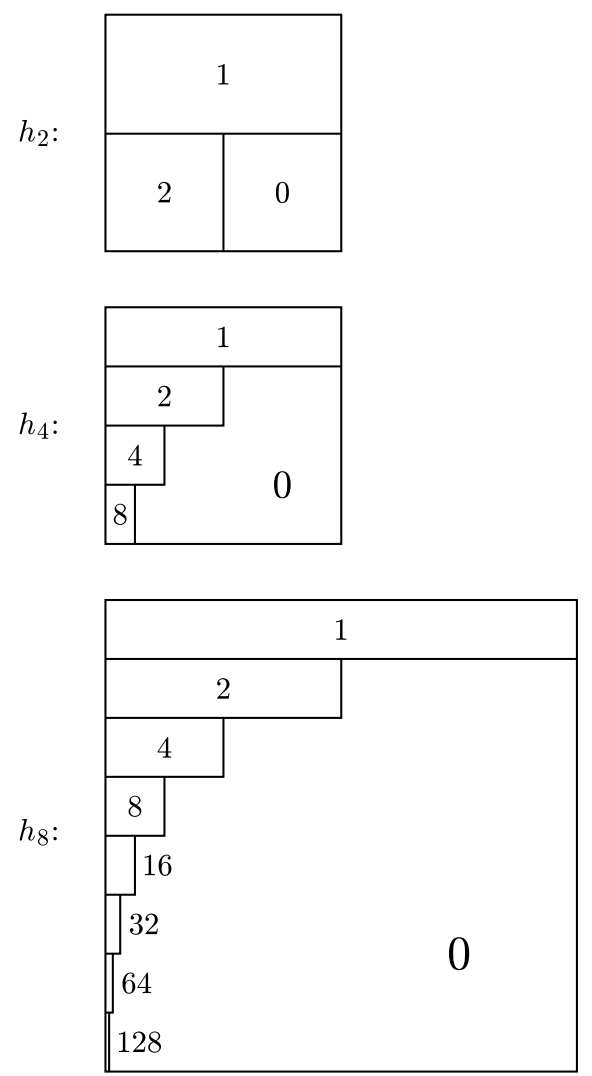

Fig. 1. Functions $h_{2}, h_{4}$, and $h_{8}$

Proof. Divide $Q$ into $2^{2 n}$ regions $I_{j, k}, j=1, \ldots, 2^{n}, k=1, \ldots, 2^{n}$, where $I_{j, k}$ is defined as follows:

$$
I_{1, k}=\left[0,2^{-2^{n}+1}\right] \times\left[(k-1) \cdot 2^{-n}, k \cdot 2^{-n}\right], \quad 1 \leq k \leq 2^{n} ;
$$

$I_{j, k}=\left[2^{-2^{n}+j-1}, 2^{-2^{n}+j}\right] \times\left[(k-1) \cdot 2^{-n}, k \cdot 2^{-n}\right], \quad 2 \leq j \leq 2^{n}, 1 \leq k \leq 2^{n}$.

Let $\widetilde{I}_{j, k}=\int_{I_{j, k}} M_{y} h_{2^{n}}$.

Note that, in $Q, M_{y} h_{2^{n}}(u, v)$ is nonincreasing in $u$ for any fixed $v \in$ $[0,1]$. Note also that if $(u, v) \in I_{j, k}^{\circ}$ and $(w, v) \in I_{j, k}^{\circ}$, then $M_{y} h_{2^{n}}(u, v)=$ $M_{y} h_{2^{n}}(w, v)$.

Now, if $(u, v) \in I_{1, k}$, then $M_{x} M_{y} h_{2^{n}}(u, v)=M_{y} h_{2^{n}}(u, v)$. Hence

$$
\int_{I_{1, k}} M_{x} M_{y} h_{2^{n}}(u, v) d u d v=\widetilde{I}_{1, k}, \quad 1 \leq k \leq 2^{n} .
$$


Suppose $2 \leq j \leq 2^{n}$. Then

(9)

$$
\begin{aligned}
& \int_{I_{j, k}} M_{x} M_{y} h_{2^{n}}=\int_{I_{j, k}} \frac{1}{x}\left[\int_{0}^{x} M_{y} h_{2^{n}}(u, v) d u\right] d v d x \\
= & \int_{I_{j, k}} \frac{1}{x}\left[\frac{\widetilde{I}_{1, k}+\ldots+\widetilde{I}_{j-1, k}}{2^{-n}}+\int_{2^{-2^{n}+j-1}}^{x} M_{y} h_{2^{n}}(u, v) d u\right] d v d x \\
= & \left(\widetilde{I}_{1, k}+\ldots+\widetilde{I}_{j-1, k}\right) \log 2+\int_{I_{j, k}} \frac{1}{x} \int_{2^{-2^{n}+j-1}}^{x} M_{y} h_{2^{n}}(u, v) d u d v d x \\
= & \left(\widetilde{I}_{1, k}+\ldots+\widetilde{I}_{j-1, k}\right) \log 2+\int_{I_{j, k}} \frac{1}{x}\left(M_{y} h_{2^{n}}(x, v)\right)\left(x-2^{-2^{n}+j-1}\right) d v d x \\
= & \left(\widetilde{I}_{1, k}+\ldots+\widetilde{I}_{j-1, k}\right) \log 2+\int_{I_{j, k}} M_{y} h_{2^{n}}\left(x_{0}, v\right)\left(1-\frac{2^{-2^{n}+j-1}}{x}\right) d v d x \\
& \quad\left(\text { where } x_{0} \text { is an arbitrary element of }\left(2^{-2^{n}+j-1}, 2^{-2^{n}+j}\right)\right)
\end{aligned}
$$$$
=\left(\widetilde{I}_{1, k}+\ldots+\widetilde{I}_{j-1, k}\right) \log 2+\widetilde{I}_{j, k}
$$$$
-(\log 2)\left(2^{-2^{n}+j-1}\right)\left(\int_{(k-1) \cdot 2^{-n}}^{k \cdot 2^{-n}} M_{y} h_{2^{n}}\left(x_{0}, v\right) d v\right)
$$$$
=\left(\widetilde{I}_{1, k}+\ldots+\widetilde{I}_{j-1, k}\right) \log 2+\widetilde{I}_{j, k}-\widetilde{I}_{j, k} \cdot \log 2 .
$$

So,

$$
\begin{aligned}
(10) & \int_{Q} M_{x} M_{y} h_{2^{n}}=\sum_{j, k=1}^{2^{n}} \int_{I_{j, k}} M_{x} M_{y} h_{2^{n}} \\
= & \widetilde{I}_{1,1}+\ldots+\widetilde{I}_{1,2^{n}} \\
& +\left(\widetilde{I}_{1,1}+\ldots+\widetilde{I}_{1,2^{n}}\right) \log 2+\left(\widetilde{I}_{2,1}+\ldots+\widetilde{I}_{2,2^{n}}\right)-\left(\widetilde{I}_{2,1}+\ldots+\widetilde{I}_{2,2^{n}}\right) \log 2 \\
& +\left(\widetilde{I}_{1,1}+\ldots+\widetilde{I}_{1,2^{n}}+\widetilde{I}_{2,1}+\ldots+\widetilde{I}_{2,2^{n}}\right) \log 2+\left(\widetilde{I}_{3,1}+\ldots+\widetilde{I}_{3,2^{n}}\right) \\
& -\left(\widetilde{I}_{3,1}+\ldots+\widetilde{I}_{3,2^{n}}\right) \log 2+\ldots \\
& +\left(\widetilde{I}_{1,1}+\ldots+\widetilde{I}_{1,2^{n}}+\ldots+\widetilde{I}_{2^{n}-1,1}+\ldots+\widetilde{I}_{2^{n}-1,2^{n}}\right) \log 2 \\
& +\left(\widetilde{I}_{2^{n}, 1}+\ldots+\widetilde{I}_{2^{n}, 2^{n}}\right)-\left(\widetilde{I}_{2^{n}, 1}+\ldots+\widetilde{I}_{2^{n}, 2^{n}}\right) \log 2 \\
= & \left(\widetilde{I}_{1,1}+\ldots+\widetilde{I}_{1,2^{n}}\right)+\left(\widetilde{I}_{2,1}+\ldots+\widetilde{I}_{2,2^{n}}\right)+\ldots+\left(\widetilde{I}_{2^{n}, 1}+\ldots+\widetilde{I}_{2^{n}, 2^{n}}\right) \\
& +\left(2^{n}-1\right)\left(\widetilde{I}_{1,1}+\ldots+\widetilde{I}_{1,2^{n}}\right) \log 2
\end{aligned}
$$




$$
\begin{aligned}
& +\left(2^{n}-3\right)\left(\widetilde{I}_{2,1}+\ldots+\widetilde{I}_{2,2^{n}}\right) \log 2 \\
& +\left(2^{n}-4\right)\left(\widetilde{I}_{3,1}+\ldots+\widetilde{I}_{3,2^{n}}\right) \log 2+\ldots \\
& +1 \cdot\left(\widetilde{I}_{2^{n}-2,1}+\ldots+\widetilde{I}_{2^{n}-2,2^{n}}\right) \log 2 \\
& +0 \cdot\left(\widetilde{I}_{2^{n}-1,1}+\ldots+\widetilde{I}_{2^{n}-1,2^{n}}\right) \log 2 \\
& +(-1)\left(\widetilde{I}_{2^{n}, 1}+\ldots+\widetilde{I}_{2^{n}, 2^{n}}\right) \log 2 .
\end{aligned}
$$

Proposition 9. If $1 \leq j \leq 2^{n}$, then $\widetilde{I}_{j, 1}+\ldots+\widetilde{I}_{j, 2^{n}} \sim n / 2^{n}$.

Proof. Suppose $2 \leq j \leq 2^{n}$. Then

$$
\begin{aligned}
\widetilde{I}_{j, 1}+\ldots+\widetilde{I}_{j, 2^{n}} & =\int_{2^{-2^{n}+j-1}}^{2^{-2^{n}+j}} \int_{0}^{1} M_{y} h_{2^{n}}(x, z) d z d x \\
& =2^{-2^{n}+j-1} \int_{0}^{1} M_{y} h_{2^{n}}\left(2^{-2^{n}+j-1 / 2}, z\right) d z \\
& \sim 2^{-2^{n}+j-1} \cdot \alpha_{j},
\end{aligned}
$$

where $\alpha_{j}$ satisfies (by the fact that $\int_{0}^{1} M_{y} h_{2^{n}}(u, z) d z \sim\left\|h_{2^{n}}(u, \cdot)\right\|_{L \log L}$ ) the equation

$$
\begin{aligned}
\frac{1}{2^{n}}\left[\frac{1}{\alpha_{j}} \log \left(3+\frac{1}{\alpha_{j}}\right)+\frac{2}{\alpha_{j}}\right. & \log \left(3+\frac{2}{\alpha_{j}}\right) \\
& \left.+\ldots+\frac{2^{2^{n}-j}}{\alpha_{j}} \log \left(3+\frac{2^{2^{n}-j}}{\alpha_{j}}\right)\right]=1 .
\end{aligned}
$$

Now,

$$
\begin{aligned}
\frac{1}{\alpha_{j}} \log (3 & \left.+\frac{1}{\alpha_{j}}\right)+\ldots+\frac{2^{2^{n}-j}}{\alpha_{j}} \log \left(3+\frac{2^{2^{n}-j}}{\alpha_{j}}\right) \\
& \leq \frac{1}{\alpha_{j}} \log \left(3+\frac{2^{2^{n}-j}}{\alpha_{j}}\right)+\ldots+\frac{2^{2^{n}-j}}{\alpha_{j}} \log \left(3+\frac{2^{2^{n}-j}}{\alpha_{j}}\right) \\
& \leq \frac{2^{2^{n}-j+1}}{\alpha_{j}} \log \left(3+\frac{2^{2^{n}-j}}{\alpha_{j}}\right) \\
& \leq 2\left[\frac{1}{\alpha_{j}} \log \left(3+\frac{1}{\alpha_{j}}\right)+\ldots+\frac{2^{2^{n}-j}}{\alpha_{j}} \log \left(3+\frac{2^{2^{n}-j}}{\alpha_{j}}\right)\right] .
\end{aligned}
$$

So $\alpha_{j} \sim \widetilde{\alpha}_{j}$, where $\widetilde{\alpha}_{j}$ is defined by

$$
\frac{2^{2^{n}-j+1}}{\widetilde{\alpha}_{j}} \log \left(3+\frac{2^{2^{n}-j}}{\widetilde{\alpha}_{j}}\right)=2^{n} .
$$


Then for $2 \leq j, k \leq 2^{n}$ we have

$$
\widetilde{I}_{j, 1}+\ldots+\widetilde{I}_{j, 2^{n}} \sim \widetilde{I}_{k, 1}+\ldots+\widetilde{I}_{k, 2^{n}}
$$

since

$$
\begin{aligned}
\widetilde{I}_{j, 1}+\ldots+\widetilde{I}_{j, 2^{n}} & \sim 2^{-2^{n}+j-1} \cdot \widetilde{\alpha}_{j}=2^{-2^{n}+j-1} \cdot 2^{k-j} \cdot \widetilde{\alpha}_{k} \\
& =2^{-2^{n}+k-1} \cdot \widetilde{\alpha}_{k} \sim \widetilde{I}_{k, 1}+\ldots+\widetilde{I}_{k, 2^{n}} .
\end{aligned}
$$

Clearly $\widetilde{I}_{1,1}+\ldots+\widetilde{I}_{1,2^{n}} \sim \widetilde{I}_{2,1}+\ldots+\widetilde{I}_{2,2^{n}}$, so (15) holds for $1 \leq j, k \leq 2^{n}$. As

$$
\widetilde{I}_{2^{n}, 1}+\ldots+\widetilde{I}_{2^{n}, 2^{n}}=\frac{1}{2}\left(2^{-n}+\int_{2^{-n}}^{1} \frac{2^{-n}}{x} d x\right)=2^{-(n+1)}\left(1+\log 2^{n}\right) \sim n / 2^{n},
$$

we get the assertion.

We now finish the proof of Lemma 8. The proposition and (10) imply

$$
\begin{aligned}
\int_{Q} M_{x} M_{y} h_{2^{n}} & \sim \frac{n}{2^{n}} \cdot 2^{n}+\left(2^{n}-2+2^{n}-3+\ldots+1\right) \cdot \frac{n}{2^{n}} \cdot \log 2 \\
& =n+\frac{1}{2}\left(2^{n}-1\right)\left(2^{n}-2\right) \cdot \frac{n}{2^{n}} \cdot \log 2 \\
& \sim n+\frac{1}{2} \cdot \log 2 \cdot n \cdot 2^{n} \sim n \cdot 2^{n} .
\end{aligned}
$$

Lemma 10.

$$
\int_{Q} M_{y} M_{x} h_{2^{n}} \leq 10 \cdot 2^{n}
$$

Proof. Clearly if $(u, v) \in Q$, then

$$
M_{x} h_{2^{n}}(u, v) \leq \begin{cases}2^{2^{n}-1}, & u \leq 1 / 2^{2^{n}-1} \\ 1 / u, & u>1 / 2^{2^{n}-1}\end{cases}
$$

So

$$
M_{y} M_{x} h_{2^{n}}(u, v) \leq \begin{cases}2^{2^{n}-1}, & u \leq 1 / 2^{2^{n}-1} \\ 1 / u, & u>1 / 2^{2^{n}-1}\end{cases}
$$

Then

$$
\int_{Q} M_{y} M_{x} h_{2^{n}} \leq 2^{2^{n}-1}\left(\frac{1}{2^{2^{n}-1}}\right)+\int_{2^{1-2^{n}}}^{1} \frac{1}{x} d x=1+\log \left(2^{2^{n}-1}\right) \leq 10 \cdot 2^{n} .
$$

We now define the function $h$ by

$$
h=\sum_{k=1}^{\infty} \frac{1}{2^{k+1}} \cdot \frac{1}{2^{4^{k-1}}} h_{2^{4^{k-1}}} .
$$


Then

$$
\begin{aligned}
\int_{Q} M_{y} M_{x} h & \leq \sum_{k=1}^{\infty} \frac{1}{2^{k+1}} \cdot \frac{1}{2^{4^{k-1}}} \int_{Q} M_{y} M_{x} h_{2^{4^{k-1}}} \\
& \leq 10 \sum_{k=1}^{\infty} \frac{1}{2^{k+1}} \cdot \frac{1}{2^{4^{k-1}}} \cdot 2^{4^{k-1}}=5
\end{aligned}
$$

but

$$
\begin{aligned}
\int_{Q} M_{x} M_{y} h & \geq \lim _{k \rightarrow \infty} \frac{1}{2^{k+1}} \cdot \frac{1}{2^{4^{k-1}}} \int_{Q} M_{x} M_{y} h_{2^{4^{k-1}}} \\
& \sim \lim _{k \rightarrow \infty} \frac{1}{2^{k+1}} \cdot \frac{1}{2^{4^{k-1}}} \cdot 2^{4^{k-1}} \cdot 4^{k-1}=\lim _{k \rightarrow \infty} 2^{k-3}=\infty
\end{aligned}
$$

We now show that if $f$ is a function such that $\int_{Q} M_{y} M_{x} f<\infty$ but $\int_{Q} M_{x} M_{y} f=\infty$, then there exists a set $A$ of finite measure in $\mathbb{R}^{2}$ such that $\int_{A} M_{\mathrm{S}} f=\infty$. This result is particularly interesting in view of the fact that M. E. Gomez has constructed a function $g$ supported on $Q$ such that $\int_{Q} M_{x} M_{y} g$ and $\int_{Q} M_{y} M_{x} g$ are infinite, but $M_{\mathrm{S}} g$ is integrable over every set of finite measure in $\mathbb{R}^{2}$ (see [5]). Such a construction is also implicit in the work of Bagby and Jawerth and Morrow ([1], [9]).

THEOREM 11. Suppose that $f$ is a measurable function supported on $Q$, $\int_{Q} M_{x} M_{y} f=\infty$, and $\int_{Q} M_{y} M_{x} f<\infty$. Then there exists a set $A$ of finite measure in $\mathbb{R}^{2}$ such that $\int_{A} M_{\mathrm{S}} f=\infty$.

Proof. We first recall a theorem due to Fava, Gatto, and Gutiérrez.

TheOREM 12 ([3]). Suppose $f$ is a measurable function supported in $Q$. Then $M_{x} M_{y} f$ is integrable over every set of finite measure in $\mathbb{R}^{2}$ if and only if $\|f\|_{L(\log L)^{2}(Q)}<\infty$.

The maximal operators $M, \bar{M}$, defined as follows, will be very useful to us:

Definition 13. Let $f$ be a measurable function supported on $Q$. The associated maximal function $M f$ is defined on $Q$ by

$$
M f\left(p_{1}, p_{2}\right)=\sup _{x_{1}<p_{1}<x_{2}} \frac{1}{x_{2}-x_{1}} \int_{x_{1}}^{x_{2}} \int_{0}^{1}|f(x, y)| d y d x .
$$

The associated maximal function $\bar{M} f$ is defined on $Q$ by

$$
\bar{M} f\left(p_{1}, p_{2}\right)=\sup _{y_{1}<p_{2}<y_{2}} \frac{1}{y_{2}-y_{1}} \int_{0}^{1} \int_{y_{1}}^{y_{2}}|f(x, y)| d y d x .
$$


Now, we define the auxiliary functions $g$ and $h$ as follows. If $p=\left(p_{1}, p_{2}\right)$ $\in \mathbb{R}^{2}$, let

$$
g(p)=\int_{0}^{1}\left|f\left(p_{1}, y\right)\right| d y \cdot \chi_{Q}(p), \quad h(p)=\int_{0}^{1}\left|f\left(x, p_{2}\right)\right| d x \cdot \chi_{Q}(p) .
$$

Note that if $p \in \mathbb{R}^{2}$, then

$$
M_{\mathrm{S}} f(p) \geq \frac{1}{2}\left[M_{\mathrm{S}} g(p)+M_{\mathrm{S}} h(p)\right] .
$$

Since $g$ and $h$ are tensors on $Q$, also note that

$$
\begin{aligned}
& M_{\mathrm{S}} g(p)=M_{x} M_{y} g(p)=M_{y} M_{x} g(p), \\
& M_{\mathrm{S}} h(p)=M_{x} M_{y} h(p)=M_{y} M_{x} h(p) .
\end{aligned}
$$

Now, by Theorem $12, M_{x} M_{y} g$ is integrable over every set of finite measure in $\mathbb{R}^{2}$ if and only if $g \in L(\log L)^{2}(Q)$, and similarly for $h$ in place of $g$. If $\|g+h\|_{L(\log L)^{2}(Q)}=\infty$, equations (19)-(21) imply the existence of a set of finite measure in $\mathbb{R}^{2}$ over which $M_{\mathrm{S}} f$ is not integrable. So it suffices to show $\|g+h\|_{L(\log L)^{2}(Q)}=\infty$.

An application of the Fubini Theorem and Theorem 6 yields that

$$
\|g\|_{L(\log L)^{2}(Q)} \sim \int_{Q} M M f, \quad\|h\|_{L(\log L)^{2}(Q)} \sim \int_{Q} \bar{M} \bar{M} f .
$$

So it is enough to show $\int_{Q} M M f+\int_{Q} \bar{M} \bar{M} f=\infty$. As $\int_{Q} M_{x} M_{y} f \lesssim$ $\|f\|_{L(\log L)^{2}(Q)}$, we see that the proof reduces to proving the following.

THEOREM 14. Let $f$ be a measurable function supported on $Q$. Then

$$
\|f\|_{L(\log L)^{2}(Q)} \lesssim \int_{Q} M M f+\int_{Q} M_{y} M_{x} f+\int_{Q} \bar{M} \bar{M} f .
$$

Proof. It will be technically convenient to work with the dyadic analogues of the maximal operators $M_{\mathrm{HL}}, M, \bar{M}, M_{x}$, and $M_{y}$. Recall that a dyadic interval in $[0,1]$ is an interval of the form $\left[k \cdot 2^{j},(k+1) \cdot 2^{j}\right]$, where $j$ is a nonpositive integer and $k$ is a nonnegative integer such that $(k+1) \cdot 2^{j} \leq 1$. We denote the set of dyadic subintervals of $[0,1]$ by $\mathcal{I}^{\Delta}$. A dyadic square in $Q$ is a set of the form $I \times J$, where $I$ and $J$ are dyadic intervals in $[0,1]$ of the same length. We denote the set of dyadic squares in $Q$ by $\mathcal{S}^{\Delta}$. We formally define the dyadic maximal operators $M_{\mathrm{HL}}^{\Delta}, M^{\Delta}, \bar{M}^{\Delta}, M_{x}^{\Delta}$, and $M_{y}^{\Delta}$ as follows.

Definition 15. Let $f$ be a measurable function supported on $Q$. The dyadic Hardy-Littlewood maximal function $M_{\mathrm{HL}}^{\Delta} f$ is defined on $Q$ by

$$
M_{\mathrm{HL}}^{\Delta} f(p)=\sup _{p \in S \in \mathcal{S} \Delta} \frac{1}{|S|} \int_{S}|f| \text {. }
$$


The maximal function $M^{\Delta} f$ is defined by

$$
M^{\Delta} f\left(p_{1}, p_{2}\right)=\sup _{p_{1} \in I \in \mathcal{I} \Delta} \frac{1}{|I|} \int_{I}^{1}|f(x, y)| d y d x .
$$

$\bar{M}^{\Delta} f$ is defined by

$$
\bar{M}^{\Delta} f\left(p_{1}, p_{2}\right)=\sup _{p_{2} \in I \in \mathcal{I} \Delta} \frac{1}{|I|} \int_{0}^{1} \int_{I}|f(x, y)| d y d x .
$$

$M_{x}^{\Delta} f$ is defined by

$$
M_{x}^{\Delta} f\left(p_{1}, p_{2}\right)=\sup _{p_{1} \in I \in \mathcal{I}^{\Delta}} \frac{1}{|I|} \int_{I}\left|f\left(x, p_{2}\right)\right| d x .
$$

Similarly, $M_{y}^{\Delta} f$ is defined by

$$
M_{y}^{\Delta} f\left(p_{1}, p_{2}\right)=\sup _{p_{2} \in I \in \mathcal{I}^{\Delta}} \frac{1}{|I|} \int_{I}\left|f\left(p_{1}, y\right)\right| d y .
$$

If $f$ is a measurable function supported on $[0,1]$, then the dyadic HardyLittlewood maximal function $M_{\mathrm{HL}}^{\Delta} f$ is defined on $[0,1]$ by

$$
M_{\mathrm{HL}}^{\Delta} f(p)=\sup _{p \in I \in \mathcal{I} \Delta} \frac{1}{|I|} \int_{I}|f| .
$$

We will also need the following results found in [7].

Lemma $16([7])$. Let $f$ be a measurable function supported on $Q$. Then

$$
\int_{Q} M_{\mathrm{HL}} f \sim \int_{Q}\left(M_{x} f+M_{y} f\right)
$$

Moreover,

$$
\int_{Q} M_{\mathrm{HL}} f \sim \int_{Q}\left(M f+M_{y} f\right) .
$$

Lemma $17([7])$. Let $f$ be a measurable function supported on $Q$. Then

$$
\int_{Q} M^{\Delta} M_{x}^{\Delta} f \lesssim \int_{Q} M^{\Delta} M^{\Delta} f+\int_{Q} M_{y}^{\Delta} M_{x}^{\Delta} f .
$$

Furthermore,

$$
\int_{Q} M_{x} M_{x} f \lesssim \int_{Q} M M f+\int_{Q} M_{y} M_{x} f .
$$

LEMMa $18([7])$. Let $f$ be a nonnegative measurable function supported on $Q$. Let $\widetilde{f}(x, y)$ be the function supported on $Q$ which is nonincreasing in $x$ 
(i.e. $\widetilde{f}\left(x_{1}, y\right) \geq \widetilde{f}\left(x_{2}, y\right)$ whenever $0 \leq x_{1} \leq x_{2} \leq 1$ and $\left.0 \leq y \leq 1\right)$ and such that, for each $y \in[0,1], \widetilde{f}(\cdot, y)$ and $f(\cdot, y)$ are equidistributed. Then

$$
\int_{Q} M_{\mathrm{HL}} M_{y} \tilde{f} \lesssim \int_{Q} M_{\mathrm{HL}} M_{y} f .
$$

Lemma 19. Suppose $f(x, y)$ is a nonnegative measurable function supported on $Q$ which is nonincreasing in $x$. Then

$$
\int_{Q} M_{x} M_{y} f \lesssim \int_{Q} M M f+\int_{Q} M_{y} M_{x} f .
$$

Proof. As $f$ is nonincreasing in $x, \int_{Q} M M f=\int_{Q} M_{x} M_{x} f$. So, letting $f^{\prime}$ be a function supported on $Q$ such that $f^{\prime}(x, \cdot)$ and $f(x, \cdot)$ are equidistributed for each $x \in[0,1]$ and also such that $f^{\prime}(x, y)$ is nonincreasing in $y$, we see that

$$
\begin{aligned}
\int_{Q}\left(M M f+M_{y}\right. & \left.M_{x} f\right)=\int_{Q}\left(M_{x} M_{x} f+M_{y} M_{x} f\right) \\
& \sim \int_{Q} M_{\mathrm{HL}} M_{x} f \quad(\text { by Lemma 16) } \\
& \gtrsim \int_{Q} M_{\mathrm{HL}} M_{x} f^{\prime} \quad(\text { by Lemma 18) } \\
& \sim \int_{Q} M_{x} M_{x} f^{\prime}+\int_{Q} M_{y} M_{x} f^{\prime} \\
& \gtrsim \int_{Q} M_{y} M_{x} f^{\prime} \\
& =\int_{Q} M_{x} M_{y} f^{\prime} \quad \text { (since } f^{\prime} \text { is nonincreasing in both variables) } \\
& \sim \int_{Q} M_{x} M_{y} f \quad \text { (by Theorem } 6 \text { and the Fubini Theorem). - }
\end{aligned}
$$

Lemma 20. Let $B=\left[0,2^{-n}\right] \times[0,1]$ be a subset of $Q$. Suppose $f$ is a measurable function supported in $B$. Then

$$
\int_{B} M_{\mathrm{HL}} f \lesssim \int_{B}\left(M_{x}+M_{y}\right) f
$$

Proof. Let $g(x, y)=f\left(x \bmod 2^{-n}, y\right)$. As

$$
\left|\left\{p \in Q: M_{\mathrm{HL}} g(p)>\alpha\right\}\right| \geq 2^{n}\left|\left\{p \in B: M_{\mathrm{HL}} f(p)>\alpha\right\}\right|,
$$


we see that $\int_{Q} M_{\mathrm{HL}} g \geq|B|^{-1} \int_{B} M_{\mathrm{HL}} f$. As

$$
\int_{Q} M_{\mathrm{HL}} g \sim \int_{Q}\left(M_{x}+M_{y}\right) g \sim \frac{1}{|B|} \int_{B}\left(M_{x}+M_{y}\right) f,
$$

we see that $\int_{B} M_{\mathrm{HL}} f \lesssim \int_{B}\left(M_{x}+M_{y}\right) f$.

Corollary 21. Let $R$ be a rectangle supported in $Q$ of width or height one. Let $f$ be a measurable function supported on $R$. Then

$$
\frac{1}{|R|} \int_{R} M_{\mathrm{HL}} f \lesssim \frac{1}{|R|} \int_{R}\left(M_{x}+M_{y}\right) f .
$$

Proof. This follows from Lemma 20 by symmetry arguments.

LemMa 22. Suppose $f$ is a nonnegative measurable function supported on $Q$ which is nonincreasing in $x$. Then

$$
\|f\|_{L(\log L)^{2}(Q)} \lesssim \int_{Q}\left(M M f+\bar{M} \bar{M} f+M_{y} M_{x} f\right) .
$$

Proof. We assume without loss of generality that $f \in C^{\infty}(Q)$. Note that

$$
\left|\left\{p \in Q: M_{\mathrm{HL}} f(p)>\alpha\right\}\right| \leq 1000\left|\left\{p \in Q: M_{\mathrm{HL}}^{\Delta} f(p)>\alpha / 1000\right\}\right|
$$

for all $\alpha>0$. Then

$$
\int_{Q} M_{\mathrm{HL}} M_{\mathrm{HL}} f \sim \int_{Q} M_{\mathrm{HL}}^{\Delta} M_{\mathrm{HL}}^{\Delta} f
$$

Hence

$$
\begin{aligned}
\|f\|_{L(\log L)^{2}(Q)} & \sim \int_{Q} M_{\mathrm{HL}}^{\Delta} M_{\mathrm{HL}}^{\Delta} f \\
& \sim \int_{Q} \bar{M}^{\Delta} M_{\mathrm{HL}}^{\Delta} f+\int_{Q} M_{x}^{\Delta} M_{\mathrm{HL}}^{\Delta} f \quad \text { (by Lemma 16). }
\end{aligned}
$$

It is then enough to show

$$
\begin{aligned}
& \int_{Q} \bar{M}^{\Delta} M_{\mathrm{HL}}^{\Delta} f \lesssim \int_{Q}\left(M M f+\bar{M} \bar{M} f+M_{y} M_{x} f\right), \\
& \int_{Q} M_{x}^{\Delta} M_{\mathrm{HL}}^{\Delta} f \lesssim \int_{Q}\left(M M f+\bar{M} \bar{M} f+M_{y} M_{x} f\right) .
\end{aligned}
$$

To prove (i), let $\ell$ be a unit horizontal line segment through $Q$. Let $p \in \ell$. Let $B$ be a horizontal dyadic band through $Q$ such that $\bar{M}^{\Delta} M_{\mathrm{HL}}^{\Delta} f(p) \sim$ $|B|^{-1} \int_{B} M_{\mathrm{HL}}^{\Delta} f$. Let now $f_{\text {int }}=f \cdot \chi_{B}, f_{\text {ext }}=f \cdot \chi_{B^{c}}$. Now by Corollary 21 ,

$$
\frac{1}{|B|} \int_{B} M_{\mathrm{HL}}^{\Delta} f_{\mathrm{int}} \lesssim \frac{1}{|B|} \int_{B}\left(M_{x}^{\Delta}+M_{y}^{\Delta}\right) f_{\mathrm{int}} \lesssim \bar{M}^{\Delta}\left(M_{x}^{\Delta}+M_{y}^{\Delta}\right) f(p) .
$$


Also,

$$
\frac{1}{|B|} \int_{B} M_{\mathrm{HL}}^{\Delta} f_{\mathrm{ext}}=\int_{\ell} M_{\mathrm{HL}}^{\Delta} f_{\mathrm{ext}} \lesssim \int_{\ell} M_{y} M_{x} f_{\mathrm{ext}} \lesssim \int_{\ell} M_{y} M_{x} f .
$$

(The equality above holds because $M_{\mathrm{HL}}^{\Delta} f_{\text {ext }}$ is constant on vertical slices of $B$.)

So

$$
\int_{\ell} \bar{M}^{\Delta} M_{\mathrm{HL}}^{\Delta} f \lesssim \int_{\ell} \bar{M}^{\Delta}\left(M_{x}^{\Delta}+M_{y}^{\Delta}\right) f+\int_{\ell} M_{y} M_{x} f
$$

Hence

$$
\int_{Q} \bar{M}^{\Delta} M_{\mathrm{HL}}^{\Delta} f \lesssim \int_{Q} \bar{M}^{\Delta}\left(M_{x}^{\Delta}+M_{y}^{\Delta}\right) f+\int_{Q} M_{y} M_{x} f .
$$

Now $\int_{Q} \bar{M}^{\Delta}\left(M_{x}^{\Delta}+M_{y}^{\Delta}\right) f \lesssim \int_{Q} \bar{M}^{\Delta} M_{x}^{\Delta} f+\int_{Q} \bar{M}^{\Delta} M_{y}^{\Delta} f$. It is clear that $\int_{Q} \bar{M}^{\Delta} M_{x}^{\Delta} f \leq \int_{Q} M_{y} M_{x} f$. We also have

$$
\begin{aligned}
\int_{Q} \bar{M}^{\Delta} M_{y}^{\Delta} f & \lesssim \int_{Q} \bar{M} \bar{M} f+\int_{Q} M_{x} M_{y} f & \text { (by Lemma 17) } \\
& \lesssim \int_{Q} \bar{M} \bar{M} f+\int_{Q}\left(M M f+M_{y} M_{x} f\right) & \text { (by Lemma 19). }
\end{aligned}
$$

Hence

$$
\int_{Q} \bar{M}^{\Delta}\left(M_{x}^{\Delta}+M_{y}^{\Delta}\right) f \lesssim \int_{Q}\left(M M f+\bar{M} \bar{M} f+M_{y} M_{x} f\right),
$$

and thus

$$
\int_{Q} \bar{M}^{\Delta} M_{\mathrm{HL}}^{\Delta} f \lesssim \int_{Q}\left(M M f+\bar{M} \bar{M} f+M_{y} M_{x} f\right) .
$$

So (i) is proved.

We now prove (ii). Since $f$ is nonincreasing in $x$, we have $\int_{Q} M_{x}^{\Delta} M_{\mathrm{HL}}^{\Delta} f \sim$ $\int_{Q} M^{\Delta} M_{\mathrm{HL}}^{\Delta} f$. Let $\ell$ now denote a vertical unit segment in $Q$, and $B$ the part of $Q$ to the left of $\ell$. We want to show

$$
\int_{\ell} M^{\Delta} M_{\mathrm{HL}}^{\Delta} f \sim \frac{1}{|B|} \int_{B} M_{\mathrm{HL}}^{\Delta} f \lesssim \int_{\ell}\left(M M f+\bar{M} \bar{M} f+M_{y} M_{x} f\right) .
$$

Let $f_{\text {int }}=f \cdot \chi_{B}, f_{\text {ext }}=f \cdot \chi_{B^{\mathrm{c}}}$. Now

$$
\frac{1}{|B|} \int_{B} M_{\mathrm{HL}}^{\Delta} f \lesssim \frac{1}{|B|} \int_{B} M_{\mathrm{HL}}^{\Delta} f_{\mathrm{int}}+\frac{1}{|B|} \int_{B} M_{\mathrm{HL}}^{\Delta} f_{\text {ext }} .
$$

By Corollary 21 we have

$$
\frac{1}{|B|} \int_{B} M_{\mathrm{HL}}^{\Delta} f_{\mathrm{int}} \lesssim \frac{1}{|B|} \int_{B}\left(M_{x}+M_{y}\right) f_{\mathrm{int}} \lesssim M\left(M_{x}+M_{y}\right) f(p)
$$


for any $p \in \ell$. So

$$
\frac{1}{|B|} \int_{B} M_{\mathrm{HL}}^{\Delta} f_{\mathrm{int}} \lesssim \int_{\ell} M\left(M_{x}+M_{y}\right) f .
$$

Now if $p \in B$, then $M_{\mathrm{HL}}^{\Delta} f_{\text {ext }}(p) \leq M_{y} f(p)$ since $f(x, y)$ is nonincreasing in $x$ and $f_{\text {ext }}$ is supported to the right of $B$. Hence

$$
\frac{1}{|B|} \int_{B} M_{\mathrm{HL}}^{\Delta} f_{\mathrm{ext}} \lesssim \frac{1}{|B|} \int_{B} M_{y} f \lesssim M\left(M_{y} f\right)(q)
$$

for any point $q \in \ell$. So

$$
\frac{1}{|B|} \int_{B} M_{\mathrm{HL}}^{\Delta} f_{\mathrm{ext}} \lesssim \int_{\ell} M M_{y} f .
$$

Hence $\int_{\ell} M^{\Delta} M_{\mathrm{HL}}^{\Delta} f \lesssim \int_{\ell} M\left(M_{x}+M_{y}\right) f$. This implies

$$
\int_{Q} M M_{\mathrm{HL}}^{\Delta} f \lesssim \int_{Q} M\left(M_{x}+M_{y}\right) f .
$$

As $\int_{Q} M M_{x} f \sim \int_{Q} M M f$, since $f$ is nonincreasing in $x$, and

$$
\int_{Q} M M_{y} f \leq \int_{Q} M_{x} M_{y} f \lesssim \int_{Q}\left(M M f+M_{y} M_{x} f\right)
$$

by Lemma 19, we get the desired result.

We now complete the proof of Theorem 14, and hence of Theorem 11. $f$ is a measurable function supported on $Q$. Without loss of generality we assume $f$ is a nonnegative function as well. Let $\widetilde{f}(x, y)$ be a function supported on $Q$ which is nonincreasing in $x$ and such that $\widetilde{f}(\cdot, y)$ and $f(\cdot, y)$ are equidistributed for each $y \in[0,1]$. Now

$$
\begin{aligned}
\|f\|_{L(\log L)^{2}(Q)}=\|\widetilde{f}\|_{L(\log L)^{2}(Q)} & \\
& \lesssim \int_{Q}\left(M M \widetilde{f}+M_{y} M_{x} \widetilde{f}+\bar{M} \bar{M} \widetilde{f}\right) \quad(\text { Lemma 22) } \\
& \sim \int_{Q}\left(M_{x} M_{x} \tilde{f}+M_{y} M_{x} \tilde{f}\right)+\int_{Q} \bar{M} \bar{M} \widetilde{f} \\
& \sim \int_{Q} M_{\mathrm{HL}} M_{x} \tilde{f}+\int_{Q} \bar{M} \bar{M} \widetilde{f} \quad(\text { Lemma 16) } \\
& \sim \int_{Q} M_{\mathrm{HL}} M_{x} f+\int_{Q} \bar{M} \bar{M} f \quad\left(\text { as }\left\|M_{x} f\right\|_{L \log L(Q)} \sim\left\|M_{x} \widetilde{f}\right\|_{L \log L(Q)}\right)
\end{aligned}
$$




$$
\begin{aligned}
& \sim \int_{Q} M_{x} M_{x} f+\int_{Q} M_{y} M_{x} f+\int_{Q} \bar{M} \bar{M} f \quad \text { (Lemma 16) } \\
& \lesssim \int_{Q} M M f+\int_{Q} M_{y} M_{x} f+\int_{Q} \bar{M} \bar{M} f \quad \text { (Lemma 17) }
\end{aligned}
$$

as desired.

\section{References}

[1] R. J. Bagby, A note on the strong maximal function, Proc. Amer. Math. Soc. 88 (1983), 648-650.

[2] A. Córdoba and R. Fefferman, A geometric proof of the strong maximal theorem, Ann. of Math. 102 (1975), 95-100.

[3] N. A. Fava, E. A. Gatto, and C. Gutiérrez, On the strong maximal function and Zygmund's class $L\left(\log _{+} L\right)^{n}$, Studia Math. 69 (1980), 155-158.

[4] R. Fefferman, Multiparameter Fourier analysis, in: Beijing Lectures in Harmonic Analysis, Princeton Univ. Press, 1986, 47-130.

[5] M. E. Gomez, A counterexample for the strong maximal operator, Studia Math. 78 (1984), 199-212.

[6] P. A. Hagelstein, Córdoba-Fefferman collections in harmonic analysis, submitted for publication.

[7] -, Rearrangements and the local integrability of maximal functions, submitted for publication.

[8] G. H. Hardy and J. E. Littlewood, A maximal theorem with function-theoretic applications, Acta Math. 44 (1930), 81-116.

[9] B. Jawerth and G. Morrow, A note on the strong and iterated maximal operators, unpublished.

[10] B. Jessen, J. Marcinkiewicz and A. Zygmund, Note on the differentiability of multiple integrals, Fund. Math. 25 (1935), 217-234.

[11] E. M. Stein, Note on the class L $\log L$, Studia Math. 132 (1969), 305-310.

[12] - Singular Integrals and Differentiability Properties of Functions, Princeton Univ. Press, 1970.

[13] A. Zygmund, Trigonometric Series, Cambridge Univ. Press, 1959.

Department of Mathematics

Princeton University

Princeton, NJ 08544, U.S.A.

E-mail: phagelst@math.princeton.edu

Received May 24, 2000

Revised version February 2, 2001 